\title{
Le discours sur l'« art nègre » : modèle de la réception de la future littérature nègre?
}

János Riesz

\section{(2) OpenEdition}

\section{Journals}

Édition électronique

URL : http://journals.openedition.org/actesbranly/482

DOI : $10.4000 /$ actesbranly.482

ISSN : 2105-2735

Éditeur

Musée du quai Branly Jacques Chirac

Référence électronique

János Riesz, « Le discours sur l'« art nègre » : modèle de la réception de la future littérature nègre? », Les actes de colloques du musée du quai Branly Jacques Chirac [En ligne], 3 | 2011, mis en ligne le 21 avril 2011, consulté le 08 septembre 2020. URL : http://journals.openedition.org/actesbranly/482 ; DOI : https://doi.org/10.4000/actesbranly.482

Ce document a été généré automatiquement le 8 septembre 2020

(c) Tous droits réservés 


\title{
Le discours sur l'« art nègre » : modèle de la réception de la future littérature nègre?
}

\author{
János Riesz
}

1 Les faits sont connus : depuis les publications de Jean Laude (La peinture française et "l'art nègre" (1905-1914). Contribution à l'étude des sources du fauvisme et du cubisme, 1968/2006, Paris, Klincksieck) et les deux volumes édités par William Rubin (1984/1987), nous connaissons dans le détail les rapports entre la peinture françaiseet l'« art nègre », ou, comme le dit le sous-titre du livre de Jean Laude, les «sources du fauvisme et du cubisme». D'autres ouvrages collectifs - plus récents - ont réuni des études sur l'ensemble de la présence noire dans l'entre-deux-guerres, ainsi le Paris Noir (2001) et le Black Paris (2006) sur la base d'une exposition à Bayreuth. Je ne vais donc pas répéter dans le détail des faits connus depuis longtemps. L'art africain, l'« art nègre " était visible depuis le début du siècle dernier. Il fut critiqué et apprécié, voire admiré, et - surtout - collectionné. En simplifiant on peut dire que c'était à partir de l'art, la sculpture surtout, que la culture africaine entra dans l'horizon du public européen.

2 Ma question est ailleurs : quelle a été l'influence de cette découverte de l'«art nègre ", des «arts primitifs » en général, ses répercussions au moment où est apparue une littérature noire? Les premiers textes autobiographiques et romanesques des années vingt, le mouvement de la Négritude dans les années 1930 et 1940 ? Le discours sur l'art nègre a-t-il anticipé ou préfiguré le discours sur la littérature africaine ? Quels sont les rapports entre les deux discours? L'enthousiasme suscité par l'«art nègre » a-t-il préparé le chemin ou fut-il plutôt un obstacle, un empêchement à une juste appréciation de la littérature/poésie qui vient après? Quelles relations se sont établies entre les deux sphères?

3 Les manuels et autres histoires de la Littérature Nègre $(1984,1990)$ restent assez avares sur le sujet. Jacques Chevrier, dans le chapitre "Naissance d'une Littérature " (pp. 15-47) consacre une seule page à «la découverte de l'art nègre à Paris ». Nous y lisons : 
« Entre 1905 et 1907, un groupe de peintres et d'artistes parisiens, lassés des canons et des modèles traditionnels de l'art occidental, découvre pour la première fois la statuaire et le masque africain. Considérés jusque-là comme de simples curiosités, des "fétiches", ces objets deviennent en quelques années le ferment d'un art nouveau. » (p. 18)

Par la suite, les « répercussions » de cet « art nègre » sont situées dans trois domaines : (1) en peinture, chez Picasso et les cubistes ; (2) en musique, du Jazz à la Revue Nègre de Joséphine Baker ; (3) finalement, dans le domaine littéraire, influençant des auteurs français tels qu'Apollinaire, Blaise Cendrars et Philippe Soupault, influence que JeanClaude Blachère a appelée Le Modèle Nègre (1981) (que Chevrier n'évoque pas).

Rien ne nous est dit quant à une possible influence du discours sur l'art nègre sur le rapport au discours de critique littéraire postérieur. À la limite, on pourrait conjecturer un certain parallélisme : la haute estime en laquelle on tenait l'art nègre a sûrement eu des répercussions sur la perception de la littérature nègre. Mais - et c'est là ma question - n'y aurait-il eu aucun lien entre le discours concernant l'art nègre et le discours sur la littérature noire postérieure ? Je me suis surtout posé la question en ce qui concerne Carl Einstein, précurseur et pionnier d'un discours "scientifique " sur l'art nègre, avec Negerplastik (1915) publié pendant la guerre, et Afrikanische Plastik (1920) dont une version française, faite par Thérèse et Raymond Burgard (La Sculpture Africaine), parut en 1922.

6 Carl Einstein, juif allemand originaire de Karlsruhe, poète, historien et théoricien de l'art, face à l'antisémitisme ambiant en Allemagne, avait, dès 1928, choisi l'exil français, parisien, où il fréquentait les milieux des artistes depuis 1907 et avait d'excellents rapports avec les peintres de l'époque, grâce aussi à son ami Daniel-Henry Kahnweiler (également originaire de Karlsruhe), le célèbre marchand d'art. Vers la fin des années 1920, Einstein avait rejoint le groupe constitué autour de Georges Bataille et Michel Leiris et fut l'un des principaux éditeurs et auteurs de la revue Documents qui a fait paraître quinze numéros entre 1929 et 1930. Dans la guerre civile espagnole Einstein a rallié les troupes républicaines. Après son retour en France en 1939, il fut interné dans un camp français de réfugiés, et quand les Allemands occupèrent la France il tenta de fuir en direction de l'Espagne. Il mourut - comme Walter Benjamin - à la frontière espagnole en se suicidant. Après la seconde guerre mondiale il a été longtemps oublié ; c'est seulement dans les années 1980 qu'on l'a redécouvert conjointement en France et en Allemagne, avec les études remarquables de Liliane Meffre et de Klaus Kiefer (mais déjà en 1938 l'américain Robert Goldwater avait signalé le rôle de pionnier de Carl Einstein dans la « découverte » de l'art africain). À titre d'exemple voici un jugement de Liliane Meffre, germaniste et historienne de l'art, dans l'introduction de son édition bilingue de Negerplastik (1998, Paris-Montréal, L'Harmattan) :

«La Sculpture nègre de Carl Einstein compte au nombre des œuvres maîtresses du $\mathrm{xx}^{\mathrm{e}}$ siècle. Par une analyse formelle audacieuse et novatrice, cet ouvrage a, en effet, conféré aux objets d'art africain un statut définitif d'œuvres d'art à part entière. Véritable découvreur de l'art africain, Carl Einstein a pour la première fois dans l'histoire de l'art occidental porté un regard sans préjugé, sans a priori ni ethnocentrisme sur un art dit tribal, primitif. » (p.7)

La question que je me suis donc posée était : $Y$ avait-il un contact, fût-il éphémère - ou une connaissance / présence de Carl Einstein auprès des critiques et historiens français de l'«art nègre » de l'époque ? Certes, la période de la guerre et de l'après-guerre n'était pas propice à la réception d'un critique allemand dont le premier essai avait du 
mal à être compris, en France comme en Allemagne, parce que «trop aride, trop technique, trop intellectuel » (Meffre $2002:$ 115).

L'intérêt des ethnologues et des collectionneurs qui dominaient jusqu'alors le discours sur l'art nègre était tout autre. Dans le premier livre sur le sujet publié après la Grande Guerre, L'Art Nègre et l'Art Océanien, avec 40 planches hors texte, paru en 1919 aux éditions Devambez à Paris, les deux auteurs, André Level et Henri Clouzot, évoquent en passant le premier livre de Carl Einstein sans nommer l'auteur, quand ils disent : « En dehors du guide raisonné et illustré aux collections ethnographiques du British Museum [...] il n'existait récemment sur l'art sauvage, à l'exception d'un livre allemand de métaphysique transcendantale, que des ouvrages circonscrits à une contrée ou à une catégorie d'objets, tels que deux études anglaises sur le Bénin, célèbre par l'art du métal.» Donc: "un livre allemand de métaphysique transcendantale », cela ne peut que se référer à la Negerplastik (nous ne savons pas d'où les auteurs connaissaient le texte d'Einstein).

Il est évident que le but des deux auteurs fut tout autre. Ils le définissent ainsi : « Il nous plait de formuler les données les plus significatives de l'art sauvage, d'en définir les courants, d'élire, à nos risques et périls, et d'étudier les œuvres qui nous paraissent les plus achevées.» (Level et Clouzot 1919: 9) Perspective que Jean Laude qualifiera d'« intéressée " (il se réfère à leur intérêt de marchand d'art primitif) (Laude 2006 : 102). Cela vaut également pour les autres études qui sont évoquées dans l'avant-propos de Level et Clouzot : ainsi, celle de Marius de Zayas, publiée à New York en 1916, African Negro Art: its Influence on Modern Art, qu'ils caractérisent d'«étude [...] contenant d'intéressantes considérations sur la mentalité et l'esthétique des nègres " (9). Selon Jean Laude (p. 101), le livre de Zayas « reflète [...] les goûts et les tendances de l'époque. [Il] était très informé de la vie picturale française [...] les objets qu'il reproduit appartiennent en majeur partie, à des collections françaises publiques [...] ou privées. »

Il en est de même du troisième auteur que Level et Clouzot citent dans leur avantpropos : il s'agit du collectionneur - qui par la suite devint le collectionneur le plus important de l'art africain - Paul Guillaume, ami de Guillaume Apollinaire avec lequel il rédigea un album tiré à petit nombre, en 1917. En 1926 il publia un autre ouvrage sur la sculpture africaine, en collaboration avec Thomas Munro, Primitive Negro Sculpture (New York, Londres, 1926), dont l'édition française (La Sculpture Nègre Primitive) parut en 1929 (Paris, G. Crès \& Cie) - je cite d'après cette édition. Dans l'introduction de ce livre sont cités en note de bas de page les titres de Level et Clouzot, Paul Guillaume et Guillaume Apollinaire, les deux publications de Carl Einstein, la Negerplastik d'après la deuxième édition de Munich en 1920, et Afrikanische Plastik, la traduction française n'étant pas évoquée. Il est probable que la critique de ces publications dans le texte se réfère aussi - et peut-être surtout - à Carl Einstein. Je cite l'introduction du livre de Guillaume/ Munro dans sa version française :

«On chercherait en vain un exposé clair et simple de ce qui distingue la sculpture nègre des autres sculptures, des traits caractéristiques qui, en elle, doivent être admirés ou critiqués. [...] On a publié quelques brochures avec des reproductions photographiques. Chacune d'elles comporte un court texte avec quelques commentaires, mais aucun essai de discussion sérieuse sur les sculptures en tant que manifestations d'art. On a donné en bas de chaque objet le nom de la tribu ou du territoire d'où on le suppose venu ; mais on n'a tenté aucun effort pour décrire les caractéristiques de ces diverses localités. En réalité, peu d'écrivains semblent avoir eu l'idée qu'il pouvait y avoir dans la sculpture nègre des traditions et des variétés bien définies. [...] On a essayé de tous les procédés pour l'étude de la 
sculpture nègre, sauf de celui qui consiste à l'observer directement et à décrire

clairement ses caractéristiques. » (Guillaume et Munro 1929 : 12) des publications comme celles de Carl Einstein et ce qu'ils proposent à la place apparaît clairement: plus de clarté - ce qui ne signifie rien d'autre que de rester dans les paradigmes du discours habituel (je renvoie à ce que Roland Barthes et Henri Meschonnic, entre autres, ont dit par rapport à la clarté française), qu'on donne des explications et des inter-prétations "ethnographiques ». La signification d'une telle étude ethnogra-phique devient claire quand on lit - chez Guillaume/Munro - les chapitres sur «La mentalité des Noirs » (1929: 21-26), et «Types des œuvres d'art produites par les Nègres » $(1929: 26-36)$, cela ne veut rien dire d'autre qu'être conforme à la doctrine et à l'idéologie coloniale qui voit «le nègre moderne en cours de civilisation » (surtout dans les colonies françaises). Je donne une seule citation à titre d'exemple :

«Un fait général domine, incontestablement: ils n'ont développé en eux aucune culture intellectuelle durable, aucune technique de science appliquée, et sont incapables de suivre la logique d'un raisonnement abstrait » $(1929: 23)$.

Nous sommes en plein dans ce que les auteurs d'un volume sur la Culture Coloniale - La France conquise par son Empire 1871-1931 appellent « le bain colonial » (Pascal Blanchard, Sandrine Lemaire [éd.], Paris, Autrement, « Mémoires 86 », $2003: 179-190)$, un contexte où il était difficile sinon impossible de se situer en dehors du cadre colonial. Ne pas parler du fait colonial - comme le fit Carl Einstein dans Negerplastik - était donc déjà se situer en dehors de cet univers discursif (ou formation discursive selon Michel Foucault).

En ce qui concerne Carl Einstein et la revue Documents,Liliane Meffre, Klaus Kiefer et Conor Joyce ont déjà montré les injustices de la critique française envers le juif allemand, et ont protesté contre l'oubli partial et scandaleux dont Carl Einstein fut victime. Je ne reviendrai pas là-dessus. Mais je retiens ceci : "Documents: deux ans d'aventure, quinze numéros, une équipe hors du commun, hétéroclite et "impossible", forgent une sorte de monument à l'esprit des temps modernes, souvent insolite et irrévérencieux, porteur d'une nouvelle esthétique et voulant comme l'ethnographie se fonder sur des documents. » (Meffre 2002 : 233) Or, la question qui nous intéresse est de comprendre pourquoi ce «monument à l'esprit des temps modernes » et les articles de Carl Einstein n'ont laissé - à notre connaissance - aucune trace dans les débats et les écrits de la jeune intelligentsia noire dans le Paris des années 1930, et notamment dans cette autre revue qui suit de si près Documents et dont on penserait qu'elle aurait beaucoup en commun avec Documents : La Revue du Monde Noir - The Review of the Black World, revue bilingue, ce qui veut dire qu'elle voulait s'adresser en même temps aux Noirs de la diaspora des colonies françaises et à ceux de l'Amérique du Nord. Ses collaborateurs sont en majorité des Noirs et des Métis antillais, originaires de la Guadeloupe, la Martinique, Haïti, exerçant des professions libérales, des bourgeois, des étudiants.

Surtout si l'on considère que les contributions de Carl Einstein dans Documents semblent tenir compte, en quelque sorte, des critiques antérieures, des reproches qu'on lui avait faits. Il essaie d'y apporter des connaissances ethnographiques comme complément à ses considérations esthétiques (auxquelles s'était limitée la Negerplastik), dans l'introduction à l'article «À propos de l'Exposition de la Galerie Pigalle » il en fait presque un programme explicite : 
«MÉTHODE POUR L'ÉTUDE DE L'ART AFRICAIN. - On traite encore aujourd'hui l'immense sujet de l'art africain plus sommairement que l'histoire de l'art de n'importe quelle ville européenne. Pourtant, il faut traiter cet art historiquement, et non plus seulement le considérer sous le seul point de vue du goût et de l'esthétique. Que l'on rassemble les traditions variées des tribus et les différents mythes, pour former une mythologie comparée de l'Afrique. On y reconnaît une concordance approximative des traditions. On prend égard, avant tout, aux mythes des migrations qui expliquent les différentes couches culturelles et historiques de l'Afrique. On détermine les différentes œuvres d'art d'après leurs indices de tribus et avant tout le tatouage. » (Documents 2(2), 1930 : 104-110)

Voilà tout un programme de l'ethnologisation des études de l'art africain. La nouvelle orientation ne concerne pas seulement Carl Einstein lui-même. Il va plus loin encore : il associe plusieurs ethnologues et historiens de l'art moderne allemands à la revue, cherche même à appliquer les méthodes de l'ethnologie à l'étude des artistes modernes européens. Et j'ai même l'impression que les articles de Carl Einstein dans Documents sont moins hermétiques, plus transparents, plus lisibles, plus accessibles en tout cas que certains articles antérieurs en langue allemande. Est-ce en vertu de la "clarté » de la langue française ou dû simplement au fait qu'on est toujours plus précis, plus univoque quand on traduit d'une langue dans une autre? Comment se fait-il donc que, malgré ce rapprochement, l'échange entre les deux revues n'ait pas eu lieu, que la communication entre leurs auteurs ne se soit pas mise en marche? Qu'un transfert de l'une à l'autre n'ait pas eu lieu et, vu d'aujourd'hui, semble même avoir été exclu.

D'ailleurs, le seul auteur qui figure dans les deux revues - si j'ai bien vérifié - est l'ethnologue allemand Leo Frobenius qui signe un article sur «Le spiritisme dans l'intérieur de l'Afrique » dans La Revue du Monde Noir (2(5) : 275-280) et un autre sur les «Dessins rupestres du sud de la Rhodésie» dans Documents (2:185-188). Est-ce un hasard si c'est précisément Frobenius qui, à partir de la traduction française de sa Kulturgeschichte Afrikas (Histoire de la Civilisation Africaine, 1936) devint le grand éveilleur de la conscience des jeunes poètes de la Négritude, et que Carl Einstein tombe dans un oubli qui semble total?

17 Le climat intellectuel de l'époque impose certains thèmes et surtout une certaine orientation par rapport à certains thèmes, et peut-être les auteurs de Documents étaient-ils plus libres, plus « affranchis » par rapport à certains discours en vigueur que les auteurs venant pour la plupart des (anciennes) colonies françaises de La Revue du Monde Noir. Le débat y tourne autant ou plus autour de la race que de la culture. Parler de l'art nègre dans le cadre de la Revue du Monde Noir c'est situer et expliquer cet art à partir de l'appartenance raciale des artistes. Le jeune Louis-Thomas Achille (qui sera encore l'auteur de la préface de la réédition de la revue en 1992) écrit dans le premier de ses deux articles sur « L'Art et les Noirs » dans le premier numéro (57-60) de La Revue du Monde Noir :

«Les Noirs sont profondément artistes. Depuis la diffusion de la musique et des danses nègres américaines [...], c'est une qualité qui leur est universellement connue. [...] En elle [la race], l'on découvre, moins un sens esthétique, dont l'exercice demande à l'intelligence une contribution indispensable et de premier plan [...] qu'un instinct artistique, exigeant pour le corps, tout autant que pour l'âme, une satisfaction urgente et fréquente. » (italiques de l'auteur, J.R.)

18 Cette détermination raciale, physique, charnelle, à la base de sa disposition esthétique, "prédispose la race plus à la sensation qu'à la production artistique ", donc plus à la danse par exemple, "l'œuvre d'art la plus immédiatement produite » qu'à la 
sculpture, où «le nombre des productions [...] n'est pas du tout proportionné à l'étendue et à la profondeur du sens artistique de la race ». Et pareillement, dans la suite de cet article sur "L'Art et les Noirs ", dans le deuxième numéro de la Revue (98-101) Achille insiste : «Nulle race ne danse autant que la race nègre. Chez les Noirs tout finit, non pas par une chanson, mais par un bal. » (98) Et : « Après la danse vient la musique. » (99) On constate comme une réserve chez Achille face à la sculpture. Le fait même que la sculpture ait « inspiré en Afrique trop d'artistes pour que l'on ne la considère pas comme un des arts favoris des Noirs " semble, aux yeux de l'auteur, un " phénomène surprenant", car " danse et sculpture semblent en effet s'opposer comme mouvement et stabilité, chaleur et froid, vie et mort. " Comment donc concilier ces contradictions? Par un tour qui semble un peu tiré par les cheveux :

«[...] les deux arts satisfont des tendances qui sont souvent les mêmes. Goût de la forme et des lignes prodiguées par un danseur nu comme par une statuette-fétiche. [...] La sculpture satisfait en outre le sensualisme nègre en offrant aux sens une reproduction suggestive de leurs objets familiers, mieux que ne peut le faire la peinture, art traître et mensonger qui prétend nous suggérer la nature en supprimant une des dimensions de l'espace. [...] La religion vient appuyer ce sensualisme d'un animisme qui dote chaque statue d'une vie personnelle physique et morale, parachevant ainsi l'assimilation entre l'œuvre d'art et la nature. » 1930 avaient seulement le choix. La belle étude de Philippe Dewitte sur Les Mouvements Nègres en France 1919-1939 (préface de Juliette Bessis, l'Harmattan, qui date de 1985, mais qui est toujours d'actualité) décrit en détail comment, au début des années 1930, les « Nouveaux Nègres » de France avaient pris de l'assurance, certes, comment la vogue nègre était devenue un phénomène de société et que l'on pouvait croire que la reconnaissance de la culture négro-africaine - et donc de l'art nègre - était sur la bonne voie. Mais il est vrai aussi, comme le dit Philippe Dewitte, qu'à l'autre bord de la société française, avec la montée des thèmes fascisants (et des fascismes dans toute l'Europe) les tensions s'exacerbent et que «l'air du temps c'est aussi la résurgence des thèses du comte de Gobineau »; en tout cas, comme le dit Dewitte, « l'atmosphère est malsaine, il ne s'agit pas de s'endormir sur les lauriers de l'Art Nègre car le racisme revient en force et se passe à nouveau des vertus de la science » $(1985: 257)$. Non seulement on ne peut pas s'endormir sur ses lauriers, dans le combat qui s'annonce, on est même obligé de lutter avec les armes de l'adversaire, de s'abaisser - pour ainsi dire - à son niveau et d'accepter d'entrer dans sa logique: contre le racisme ambiant il faut développer ce que Jean-Paul Sartre appellera plus tard un « antiracisme raciste ».

21 Donc dans les années 1930 et jusque dans les premières années de l'après-guerre la situation est comme bloquée : les années 1930 s'annoncent plus difficiles que les années 1920 (plus exubérantes, plus libérales aussi) pour les artistes et intellectuels, qu'ils 
soient africains ou de la diaspora noire à Paris. La crise économique se fait sentir (dans la métropole comme dans les colonies), la situation politique est de plus en plus confuse, l'agression de l'Italie fasciste contre l'Éthiopie fut vécue comme un cauchemar par les Noirs en Europe; la guerre civile en Espagne, la montée du nazisme en Allemagne augmentent leur malaise - il se crée autour d'eux un climat d'angoisse qui est à l'origine de toutes sortes de «crises » qui vont de la résignation au désespoir, et qui peuvent mener jusqu'au suicide. Le seul de ces jeunes Noirs qui semble suivre son parcours - intellectuel et professionnel - dans le calme d'une vie réglée et régulière, est Léopold Senghor, élu président d'une association des étudiants de l'A.O.F. à Paris, et porte-parole du groupe. Il était arrivé à Paris en octobre 1928, interne au Lycée Louis le Grand, il fait sa licence de lettres en 1931, son diplôme d'études supérieures un an après, son mentor Blaise Diagne, député du Sénégal, obtient pour lui la nationalité française, Senghor fait son service militaire, il prépare son agrégation qu'il réussit premier Africain - en 1935. Il enseigne le Français et les langues classiques à Tours et dans la banlieue Est de Paris. Il écrit ses premiers poèmes, lit beaucoup à la recherche d'arguments et d'alliés pour le grand combat de sa vie : la Négritude dans le sens d'une réhabilitation et d'une revalorisation des cultures et des "valeurs " nègres. Depuis 1937, à la suite de deux grandes conférences il devient un personnage public, représentant de l'intelligence noire à Paris à qui semble promis un grand avenir.

En cette qualité, il est sollicité pour contribuer à un chapitre d'un volume sur L'Homme de Couleur, publié à la librairie Plon en 1939 et réédité en 1964. Le titre de son essai est : "Ce que l'homme noir apporte» (Liberté 1 - Négritude et Humanisme, Paris, Seuil : 22-38) et il l'ouvre par une longue citation de l'Histoire de la Civilisation africaine de Leo Frobenius, où celui-ci parle des civilisations du Soudan et de l'Afrique équatoriale. Je cite une seule phrase pour en donner le ton: «Je ne connais aucun peuple du Nord qui se puisse comparer à ces primitifs par l'unité de civilisation. » (22) Le but de Senghor dans ce long article n'est pas moins que de donner un panorama de ce qu'il entend par «culture » et « civilisation » africaines et leur apport à la civilisation moderne, celle du $\mathrm{xx}^{\mathrm{e}}$ siècle : il commence par "l'âme nègre ", continue en parlant de la sensibilité et l'émotivité des Noirs, leurs rapports avec la Nature, visible et invisible, l'animisme, leur sens de la religion, le culte des ancêtres, la société nègre, sa structure hiérarchique - de la famille au clan, à la tribu, le royaume, l'empire - les notions de travail et de propriété, les fondements de la politique en Afrique, la valeur de la personne et de la communauté, le rôle de la palabre pour la prise de décisions "démocratiques", et finalement la littérature et l'art, domaines privilégiés des apports nègres du $\mathrm{xx}^{\mathrm{e}}$ siècle, et là Senghor veut considérer prioritairement l'art plastique et la musique.

Senghor commence par une attaque en règle contre les surréalistes qui n'auraient pas eu, " pour le Nègre, une sympathie toujours discrète, c'est-à-dire éclairée ", et de là il arrive à une critique généralisée des arts en France à l'époque :

«Comment en aurait-il été autrement dans un monde asservi à la matière et à la raison, où l'on ne dénonce la raison que pour proclamer le primat de la matière. Car c'est bien là la cause de décadence de l'art au $\mathrm{xx}^{\mathrm{e}}$ siècle ; et les manifestes "pour l'Art français" que publie la revue Les Beaux Arts sont significatifs. Le réalisme et l'impressionnisme ne sont que les deux aspects d'une même erreur. C'est l'adoration du réel qui conduit à l'art photographique. À la limite, l'esprit se satisfait d'analyser et combiner les éléments du réel, en vue d'un jeu subtil, d'une variation sur le réel. » (1964:34) 
24 esthétique : [mais] de signifier ». Ce que Senghor explique comme suit :

\begin{abstract}
"Je choisis, parmi les arts plastiques, la sculpture, l'art le plus typique. Même la décoration des ustensiles les plus simples du mobilier populaire, loin de les détourner de leur but et d'être un vain ornement, souligne ce but. Art pratique, non pas utilitaire ; et classique en ce premier sens. Surtout art spirituel - on a dit à tort : idéaliste ou intellectuel - parce que religieux. Les sculpteurs ont pour fonction essentielle de représenter les Ancêtres morts et les génies par des statues qui soient, en même temps, symbole et habitacle. Il s'agit de faire saisir, sentir leur âme personnelle comme volonté efficace, de faire accéder au surréel.» (1964: 34, italiquesdans le texte)
\end{abstract}

J'ai le sentiment que dans ces paragraphes de Léopold Sédar Senghor il y a beaucoup d'éléments qui pourraient être rattachés à des énonciations de Carl Einstein, de la Negerplastik à la Fabrikation der Fiktionen. Mais en l'état actuel de mes recherches sur Carl Einstein je n'oserais pas encore m'y risquer. Ce qui est pourtant clair, c'est que Senghor, dans ce texte, est plus proche de Carl Einstein que de Paul Guillaume et de Thomas Munro qu'il cite pourtant dans la note 18 de la page 35. Quand on y regarde de plus près, le «rythme » par lequel Senghor définit le «style nègre » est plus proche de l'«élan vital» d'Henri Bergson que du «sang nègre » de Gobineau et ses séquelles. Senghor, dans une intuition de poète et de penseur, rejoint Einstein, sans probablement l'avoir lu.

Mais le vrai tournant, la coupure effective avec le vieux discours qui se voulait ethnographique, vient après la guerre. Le texte qui agit comme "manifeste» de la Négritude de la même manière que la Negerplastik de Carl Einstein avait pu servir comme manifeste au cubisme et à l'art nègre, c'est « Orphée Noir ", la célèbre préface de Jean-Paul Sartre à l'Anthologie de la nouvelle poésie nègre et malgache de langue française, éditée par Senghor, préface qui a à peu près la même longueur que la Negerplastik, une trentaine de pages, où Sartre définit la nouvelle poésie noire comme la seule poésie authentiquement révolutionnaire de l'époque (tout comme André Breton qui considérait Cahier d'un retour au pays natal d'aimé Césaire comme "le plus grand monument lyrique de l'époque» [1943, 1947]). Et c'est aussi le moment de la redécouverte (je ne dirais pas encore de la réhabilitation) du nom de Carl Einstein. En 1947, au mois de novembre, paraît le premier numéro de la revue Présence Africaine dont le directeur est Alioune Diop, ami de Senghor et sénégalais catholique comme lui. Dans le comité de patronage figurent les noms de Paul Rivet, Théodore Monod, Emmanuel Mounier, Michel Leiris, Albert Camus, Jean-Paul Sartre, Richard Wright et Paul Hazoumé.

Présence Africaine qui sera mensuel à partir de 1948 se fera connaître comme le forum le plus important d'un débat entre l'Afrique et l'Europe à l'époque de la décolonisation, et de la naissance d'un discours autonome africain. Dans le $3^{\mathrm{e}}$ numéro de la revue nous trouvons un article de Daniel-Henry Kahnweiler ("L'Art nègre et le cubisme ») qui peut être lu comme une synthèse des idées de Carl Einstein (telles qu'elles avaient évolué dans l'échange oral et épistolaire avec l'ami). Et ce n'est certainement pas un hasard si la première note de bas de page de cet essai renvoie à l'ami mort en 1940. C'est la note de la page 368 qui commence comme suit : « La Negerplastik de Carl Einstein, le premier livre paru sur l'Art Nègre en tant qu'art [...]».

Successivement ou parallèlement à cette - toutes proportions gardées - redécouverte de Carl Einstein, nous assistons à une revalorisation de la sculpture africaine en tant 
qu'expression de la «Négritude » et partie de «ce que l'homme noir a apporté » au siècle. Je ne choisis qu'un seul exemple : Louis-Thomas Achille, que nous connaissons déjà au travers de ses deux essais sur "L'Art Nègre " parus dans La Revue du Monde Noir de 1931-1932, et d'un article sur "L'artiste noir et son peuple" dans un numéro thématique de Présence Africaine (16 [n.s.], 1957 : 32-52) sur « L'Homme de Culture Noire et son Peuple » écrit, cette fois, sur la « Sculpture Africaine » :

«La sculpture est, de tous, celui qui apporte les plus honorables lettres de noblesse.

L'Afrique en est le fief, ainsi que nombre de musées et de collections d'Europe et

d'Amérique. Patrimoine écrasant! Devant ce trésor, le plus précieux peut-être, nous

sommes inquiets et perplexes autant qu'émerveillés. » (41)

Souvenons-nous de ce que Louis-Thomas Achille avait dit en 1931-1932 et quelle place la sculpture avait occupé à ses yeux à l'époque pour mesurer l'énorme progrès que le discours sur la sculpture africaine a accompli dans les vingt-cinq dernières années. Nous aimerions penser que Carl Einstein y était pour quelque chose. La réflexion sur l'art africain fera désormais partie du discours de la Négritude et du discours sur l'Afrique tout court. Et elle se fera en grande partie sur les pages de la revue Présence Africaine. Je ne veux pas (et ne peux pas) citer tous les articles qui traitent de l'art africain et qui font aussi souvent des parallèles avec la littérature. Je n'évoque que le numéro spécial de la revue, pour le 50 anniversaire de Présence Africaine (1947-1997),qui se base sur un colloque qui s'est tenu à Dakar en novembre 1997. Là c'est Abdou Sylla qui dans un long article (291-325) sur "Présence Africaine et art nègre " fait le bilan des cinquante premières années de la revue autour de cette thématique, et il prend pour point de départ - précisément - l'article de Daniel-Henry Kahnweiler qui le premier aurait «rétabli les faits» et qu'il cite sur plus de deux pages. Nous savons que Kahnweiler n'était pas seulement l'ami intime de Carl Einstein, mais que Einstein fut aussi son maître en matière de valorisation esthétique de la sculpture nègre et de ses rapports avec l'art moderne contemporain. Avec un retard de quarante ans, dû à une constellation historique qui n'était pas propice, le pionnier de l'analyse esthétique de la sculpture nègre que fut Carl Einstein, a trouvé sa place dans l'histoire du discours sur l'art nègre et simultanément - indirectement - dans l'ensemble de la production culturelle africaine au $\mathrm{xx}^{\mathrm{e}}$ siècle. On ne peut que regretter que son nom - sauf parmi les quelques spécialistes - soit toujours assez peu connu.

\section{AUTEUR}

\section{JÁNOS RIESZ}

Il a occupé une chaire de littérature comparée et de littératures francophones à l'Université de Bayreuth de 1979 à 2004. Il a publié un grand nombre d'articles et ouvrages dans ce domaine. Dernières publications en langue francaise : De la littérature coloniale à la littérature africaine Prétextes, contextes, intertextes, Karthala, 2007 ; « Astres et Désastres »-Histoire et récits de vie africains de la Colonie à la Postcolonie, Georg Olms, 2009. J. R. vit à Munich comme professeur émérite et continue à enseigner à la Hochschule für Philosophie (des Jésuites). 